\title{
DIAMAGNETIC STRUCTURES AS A BASIS OF QUASI-STATIONARY SLOW SOLAR WIND
}

\author{
V.G. Eselevich \\ Institute of Solar-Terrestrial Physics SB RAS, \\ Irkutsk, Russia, esel@iszf.irk.ru
}

\begin{abstract}
The results presented in this review reflect the fundamentals of the modern understanding of the slow solar wind (SW) structure along all the way from the Sun to Earth's orbit. The source of the slow quasistationary SW on the Sun is known to be coronal streamer belt and chains. The streamer belt encircles the Sun as a wave-like surface (skirt), representing a sequence of pairs of rays with increased brightness (plasma density) or two closely spaced lines of rays. The neutral line of the radial component of the global solar magnetic field goes along the belt between the rays of each of these pairs. The streamer belt extension in the heliosphere is the heliospheric plasma sheet (HPS). The detailed analysis of Wind and IMP-8 satellite data has shown that HPS parts in Earth's orbit are recorded as a sequence of diamagnetic tubes with increased density plasma and low interplanetary magnetic field. They represent an extension of rays with increased brightness of the streamer belt near the Sun. Their angular size remains the same over the entire way from the Sun to Earth's orbit. Each HPS diamagnetic tube has a fine internal structure on several scales, or fractality. In other words, the diamagnetic tube is a set of embedded dia-
\end{abstract}

\section{INTRODUCTION}

The quasi-stationary and sporadic solar wind (SW) is SW streams for which the lifetime of sources on the Sun is respectively longer (or much longer) and shorter than a day. The quasi-stationary slow SW in the absence of sporadic streams in Earth's orbit features a higher plasma density $N>(10 \pm 2) \mathrm{cm}^{-3}$ and a relatively low velocity $V \approx 250-450 \mathrm{~km} / \mathrm{s}$ as compared to the quasistationary fast SW flowing out of coronal holes whose maximum velocity $V \approx 450-800 \mathrm{~km} / \mathrm{s}$ [Borrini et al., 1981; Eselevich, Fainshtein, 1991, 1992]. Sources of the slow SW on the Sun are the streamer belt [Svalgaard, 1974] and chains [Eselevich et al., 1999; Eselevich et al., 2007], or psevdostreamers [Wang, 2007]). The mechanism of occurrence of the slow SW is still unclear. The sporadic SW is associated with coronal mass ejections (CMEs) whose nature is also under study.

Using Helios data, Stansby and Horbury, [2018] recorded local plasma densities $N>50 \%$ with a simultaneous decrease in the magnetic field strength $B$ (number density structures) in the slow SW at distances smaller or equal to 0.5 AU from the Sun. Statistical studies of 140 such structures have been carried out. They have shown that the spatial resolution of these structures is $\approx 50 \div 2000 \mathrm{Mm}$, which at the mean SW velocity $V \approx 400 \mathrm{~km} / \mathrm{s}$ corresponds to time scales $\approx 2 \div 80$ min. The total pressure (gas kinetic + magnetic) inside magnetic tubes whose angular size can vary by almost two orders of magnitude. These sequences of diamagnetic tubes that form the basis of the slow SW in Earth's orbit has a more general name - diamagnetic structures (DS). In the final part of this article, a comparative analysis of several events is made using the results of this review. It allowed us to find out the morphology and origin of the new term "SW diamagnetic plasmoids" (local enhancements of plasma density), which appeared in several articles published in 2012-2018. The analysis carried out at the end of this article has shown for the first time that $\mathrm{SW}$ diamagnetic plasmoids are a small-scale component of the fractal diamagnetic structures of slow SW considered in this review.

Keywords: solar wind, diamagnetic structures, diamagnetic plasmoids, streamer chain.

and outside the structures remains constant, which ensures their stability.

Karlsson et al. [2012; 2015] recorded density inhomogeneities at distances of $1 \mathrm{AU}$ from the Sun, called by the authors SW diamagnetic plasmoids. They were identified as local increases in plasma density $N>50 \%$ with a simultaneous decrease in the magnetic field modulus $B$ and constant velocity $V$ on the scale of $(0.1 \div 10) R_{\mathrm{E}}$ ( $R_{\mathrm{E}}$ is the Earth radius). The term " $\mathrm{SW}$ diamagnetic plasmoid" refers to the anticorrelated behavior of $N$ and $B$. The constant velocity inside and outside the plasmoids implies that they are carried away by the slow SW. The time of propagation of diamagnetic plasmoids is about 5-10 min [Karlsson et al., 2015], i.e. it is approximately consistent with time scales of the above number density structures recorded at $\leq 0.5 \mathrm{AU}$ [Stansby, Horbury, 2018].

Parkhomov et al. [2017, 2018] have introduced the concept of diamagnetic structures (DS) for the quasistationary slow SW flowing in the streamer belt and chains. It has a broader meaning than that of diamagnetic plasmoids. This concept arose from the results obtained in [Eselevich, Eselevich, 2006a, b], where the streamer belt has been shown to be a sequence of rays with increased brightness (plasma density). Each of these rays exhibits an increase in $N$ and a decrease in $B$, i.e. diamagnetism of rays. The rays maintain their angu- 
lar size $\approx 2-3^{\circ}$ up to $1 \mathrm{AU}$. Inside these rays, diamagnetic structures with smaller angular sizes were detected.

The main purpose of this work is to carry out a comparative analysis based on the results of this review in order to understand the origin of the term "SW diamagnetic plasmoid" used in a number of articles in the last decade.

\section{STRUCTURE OF STREAMER BELT AND CHAINS FROM THE SUN TO EARTH'S ORBIT}

The streamer belt and chains are seen in white light as a series of radially oriented coronal rays with increased brightness. This is clearly shown in Figure 1, which gives typical examples of distributions of whitelight corona brightness with background subtracted $\Pi(\Lambda, R)=P(\Lambda, R)-P_{\mathrm{S}}(\Lambda, R)$, which are constructed using LASCO $\mathrm{C} 2 / \mathrm{SOHO}$ data for two most simple limiting cases: 1) a part of the streamer belt stretched along the latitude $\Lambda$ and located practically in the plane of the sky (Figure 1, top and middle panels representing the northern and southern hemispheres of the Sun for January $12,2000) ; 2$ ) a part of the streamer belt perpendicular to the plane of the sky (bottom image for May 05,1996).

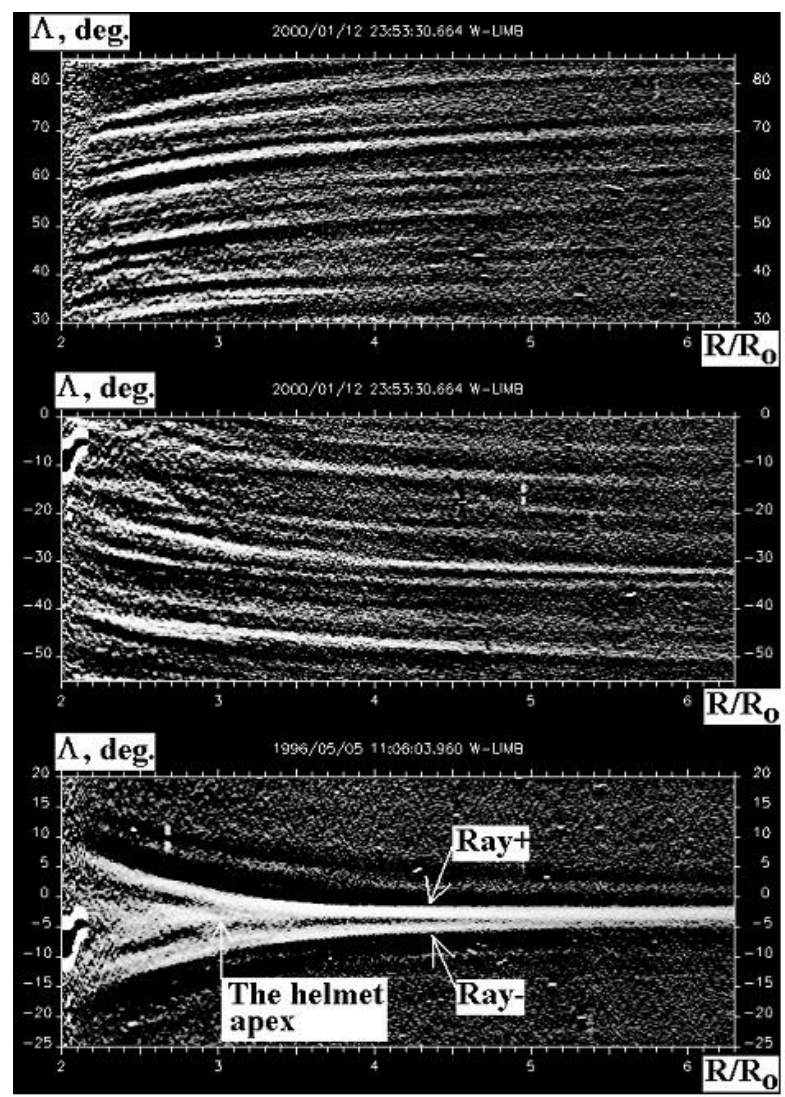

Figure 1. Brightness distributions with the background subtracted $\Pi(\Lambda, R)=\mathrm{P}(\Lambda, R)-P_{\mathrm{S}}(\Lambda, R)$ representing the averaging of $P(\Lambda, R)$ over the angular size $\delta \Lambda=5^{\circ}$ in polar coordinates $(\Lambda, R)$ in two most simple and specific cases: parts of the streamer belt in the northern and southern hemispheres of the Sun respectively, located near the plane of the sky (December $12,2000,20: 31$ UT) in the $W$ limb (top and middle panels); a part of the streamer belt perpendicular to the plane of the sky (May 05, 1996, 11:06 UT) in the $W$ limb (bottom panel). LASCO C2 data
In these examples, the background brightness $P_{\mathrm{S}}(\Lambda, R)$ is the brightness $P(\Lambda, R)$ averaged over the angular size $\delta \Lambda=5^{\circ}$.

Features of the structures of the streamer belt and chains from the Sun to Earth, which have been obtained in particular from the analysis of LASCO C2 data, are presented in Figure 2 and are as follows.

1. Streamer belt at distances $R>3 R_{\odot}\left(R_{\odot}\right.$ is the solar radius) from the center of the Sun (i.e. above the helmet apex, marked so in the bottom panel) in the absence of $\mathrm{CME}$ is a sequence of pairs of rays (or, more precisely, two closely spaced lines of rays) with increased brightness.

The angular size of all the rays is approximately the same $\left(d \approx 2-3^{\circ}\right)$; the distance between the rays along the streamer belt is $5-10^{\circ}$ [Eselevich, Eselevich, 2006a, b]. From now on, the angular size is given in the heliocentric coordinate system. The sequence of these rays is seen in the top and middle panels; and the cross-section
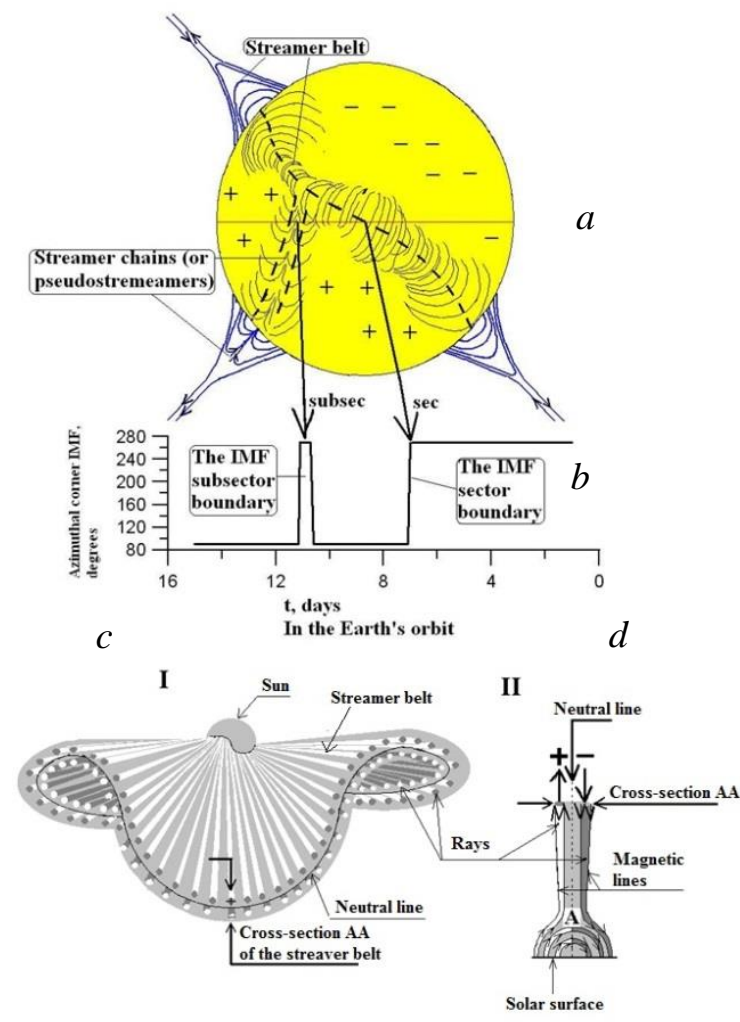

Figure 2. Scheme of magnetic field lines at bases of the coronal streamer belt and chains, separating regions on the solar surface with oppositely and equally directed radial component of the global solar magnetic field respectively $(a)$. The single dashed line is the neutral line (NL) of the radial magnetic field component passing through apices of the arches. The double dashed line shows two neutral lines along double magnetic arches at the base of the streamer chains. Scheme of distribution of the azimuth angle of the interplanetary magnetic field (IMF) in Earth's orbit when the Sun rotates along the horizontal line (ecliptic) in panel $a(b)$; ray structure of coronal streamers in space (c); cross-section (AA) of the streamer belt $(d)$. In light rays with a dark cross-section of the line above NL of the streamer belt, the magnetic field is antisunward (+); in dark rays with a dark cross-section of the line below NL it is sunward (-). The solid line is the neutral line between the rays. 
of these two lines (Ray+ and Ray-) enveloping the helmet on both sides at $R<3 R_{0}$, in the bottom panel. The magnetic field along the boundary of the helmet is an arch whose two footpoints are anchored in the photosphere [Eselevich et al., 1999]. Ray brightnesses in each pair may generally differ, and the magnetic field direction in them is opposite. This means that NL of the radial component of the global solar magnetic field passes through apices of the helmet along the belt between the rays of each pair of these two lines. Figure 2, $a$ shows the helmet as a sequence of single magnetic arches through apices of which NL passes. In the general case, the cross-section of the helmet may be composed of an odd number of magnetic field arches (one or three).

2. Rays at $10 R_{\odot}>R>(4 \div 5) R_{\odot}$ remain radial up to $\pm 1.5^{\circ}$. The lifetime of the most long-lived rays may exceed 10 days [Eselevich et al., 2001]. Quasi-stationary, i.e. averaged over fluctuations of the white-light corona brightness with periods less than one day, ray structure of the streamer belt has always existed: it sometimes decays under the action of CME and then recovers within 1-2 days [Illing, Hundhausen, 1986]. In the streamer belt, the quasi-stationary slow SW forms and propagates from the Sun.

3. Inside the streamer belt, the plasma density in rays may exceed that between rays by tens of percent. In the direction transverse to the plane of the streamer belt, $N$ can decrease several times [Howard et al., 1975].

4. White-light corona images owing to the limited spatial resolution of current coronagraphs do not allow us to observe the internal structure of higher brightness rays. The use of the radio translucence method made it, however, possible to detect small-scale structures with sizes to several angular minutes at distances $(14 \div 77) R_{\odot}$ inside a ray of the streamer belt with an angular size of $1.8^{\circ}$ [Woo et al., 1995].

5. The streamer belt encircles the Sun as a wave-like surface (skirt) (Figure 2,c). At solar minimum, the surface of the belt is close to the solar equatorial plane and has several points (two or four) of intersection with it.

The streamer belt extension in the heliosphere (slow SW) is also called heliospheric plasma sheet (HPS) [Winterhalter et al., 1994]. The streamer belt NL inside HPS in Earth's orbit manifests itself as a sector boundary of IMF (indicated by the arrow labeled sec in Figure $2, a, b$ ) separating the extended $\mathrm{SW}$ areas (of the order of tens of degrees in the heliocentric coordinate system or, accordingly, several days) with opposite signs of IMF [Korzhov, 1977]. The positive antisunward direction corresponds to the azimuth angle $\Phi<180^{\circ}$; the negative one, to $\Phi>180^{\circ}$. There is therefore an odd number of reversals of sign of IMF (if it is greater than unity) in the structure of the sector boundary.

6 . The streamer chains (or pseudostreamers) in the white-light corona appear, similar to the streamer belt, as a sequence of pairs of rays with increased brightness (density). In them, the slow SW has approximately the same properties as in the streamer belt. The streamer chains, however, differ from the belt in the fact that they separate coronal regions with open magnetic field lines having the same polarity. The cross-section of the hel- met, located at the base of the streamer chains, represents, therefore, two arches (in general, an even number of arches) [Eselevich et al., 1999]. The sequences of apices of these pairs of arches are shown by two parallel dashed curves in Figure 2, $a$.

7. In Earth's orbit, the streamer chains are recorded as areas with increased plasma density containing an even number of IMF sign changes, called subsector boundaries (the arrow labeled subsec in Figure 2, $a, b$ ) [Ivanov et al., 2002].

8. In HPS, the angular dimensions and relative changes in density observed near the Sun in ray structures of the streamer belt remain constant [Eselevich, Eselevich, 2006a, b]. In Earth's orbit, HPS has the following parameters and features [Borrini et al., 1981; Eselevich, Eselevich, 2006b]:

- relatively low SW velocity nearly constant across the sheet $V \approx(250 \div 450) \mathrm{km} / \mathrm{s}$;

- lower proton temperature $T_{\mathrm{p}}<10^{5} \mathrm{~K}$;

- higher plasma density with maximum values $N_{\max }>(10 \pm 2) \mathrm{cm}^{-3}$

- anticorrelation between variations in $N(t)$ and $B(t)$ on time scales at least of the order of hours or less;

- presence of one or several IMF sign changes representing a sector boundary or its structure (in the case of streamer chains, the number of sign changes in IMF is even, i.e. there is no sector boundary in Earth's orbit).

The presence of all these features is required for unambiguous identification of the slow SW flowing in HPS in Earth's orbit.

Since HPS is a quasi-stationary structure rotating together with the Sun, there is the following relationship between time and angular dimensions in Earth's orbit: 1 day $\approx 13.3^{\circ}$.

Following [Eselevich, Eselevich, 2006b], we introduce the term of horizontal and tilted HPS parts. It is determined from the tilt angle $\lambda$ of its associated nearSun part of NL of the radial component of the global magnetic field with respect to the solar equator. To construct the profile of NL calculated in a potential approximation at $R=2.5 R_{\odot}$ from the center of the Sun, we have used data from [http://wso.stan-ford.edu/]. Let us refer to the HPS part making an angle with the plane of the ecliptic $\lambda<10^{\circ}$ as horizontal; and with $\lambda>10^{\circ}$, as tilted. The horizontal HPS part is subject to minor distortions when its constituting slow SW moves from the Sun to Earth because it does not meet the fast SW flowing from coronal holes. At the same time, the tilted HPS part due to collision with the fast SW is often subject to additional compression (the plasma density and magnetic field increase in it); the higher is $\lambda$, the stronger is the compression [Eselevich, Fainshtein, 1991].

This picture of correspondence between the HPS parts at $1 \mathrm{AU}$ and their sources on the Sun has been examined in more detail in [Eselevich et al., 1999; Eselevich, Eselevich, 2006a, b]. This makes it possible to define DS as a basis of the slow SW. For this purpose, we analyze the spatial structure of HPS in Earth's orbit, using data with high temporal resolution from two spacecraft: Wind and IMP-8. When measured by Wind, IMF is $\approx 3 \mathrm{~s}$; by IMP-8, $\approx 12 \mathrm{~s}$. 


\section{METHOD FOR CALCULATING THE TIME OF ARRIVAL OF STREAMER BELT STRUCTURES AT EARTH'S ORBIT}

The time $T_{\text {orb }}$ of arrival of this part of the streamer belt at Earth's orbit, if this part is observed on the west limb at $T_{\mathrm{W}}$ or on the east limb at $T_{\mathrm{E}}$, is determined from the following formulas

$T_{\text {orb }}=T_{\mathrm{W}}-7$ days $+\Delta T ; T_{\text {orb }}=T_{\mathrm{E}}+7$ days $+\Delta T,(1)$ where

$$
T_{\mathrm{W}}-7 \text { days } \approx T_{\mathrm{E}}+7 \text { days }
$$

is the time when this steamer belt part passes through the central meridian.

Here, 7 days is the time of movement of this part from the limb to the central meridian (or vice versa).

The value $\Delta T$ [Eselevich, Eselevich, 2006b] found from the formula

$$
\Delta T(\mathrm{hr})=\left(4.6 \cdot 10^{4}\right) / V(\mathrm{~km} / \mathrm{s})
$$

is the time of passage of the slow SW from the Sun to Earth's orbit, $V$ is the SW velocity in Earth's orbit. For $V=350 \mathrm{~km} / \mathrm{s} \Delta T \approx 5.5$ days. From 1996 to $1999, \sim 40$ regions of the quasi-stationary slow SW were analyzed. These regions in Earth's orbit are characterized by the parameters and features mentioned above. For all the regions, $V=350 \mathrm{~km} / \mathrm{s}$ up to several tens of $\mathrm{km} / \mathrm{s}$. Therefore, when calculating $T_{\text {orb }}, \Delta T$ is everywhere set to 5.5 days.

\section{SELECTION AND ANALYSIS OF EVENTS ON DIFFERENT TIME (SPATIAL) SCALES}

As the events to study we have selected HPS parts in the absence of sporadic SW streams from measurements of SW parameters in Earth's orbit in accordance with the requirements listed above. The correspondence between the selected HPS part and the streamer belt near the solar surface in the E and $\mathrm{W}$ limbs was found using Formulas (1)-(3) in the assumption that SW parameters of this streamer belt part vary slightly during $\approx 12-14$ days.

September 4, 1996 event (day 248)

To this part near the Sun corresponds a tilted part of the streamer belt with $\lambda>10^{\circ}$. Figure 3 shows $N(t)$ and $V$ $(t)$ of SW plasma, IMF parameters averaged over $60 \mathrm{~s}$ : $B(t), B_{x}(t), B_{y}(t), B_{z}(t)$, and $\Phi(t)$. The profile of $\Phi(t)$ at $t \approx 9 \mathrm{hr}$ exhibits the simple sector boundary corresponding to the change in $\Phi$ from $\approx 135^{\circ}$ to $\approx 315^{\circ}$, which is equivalent to the change in sign of the IMF radial component from "+" (from the Sun) to "-" (to the Sun). In the general case, the sector boundary can be complex and consist of an odd number of IMF sign changes.

A question arises as to if there are time variations in $N$ and $B$ or moving quasi-static spatial structures. Note first of all that in a magnetized plasma magnetic field oscillations of two basic types can occur which carry away most energy during the development of instability: Alfvén and magnetosonic. In the Alfvén oscillations, $N=$ const, $B$ changes; in the magnetosonic ones, $N(t)$ correlates with $B(t)$.

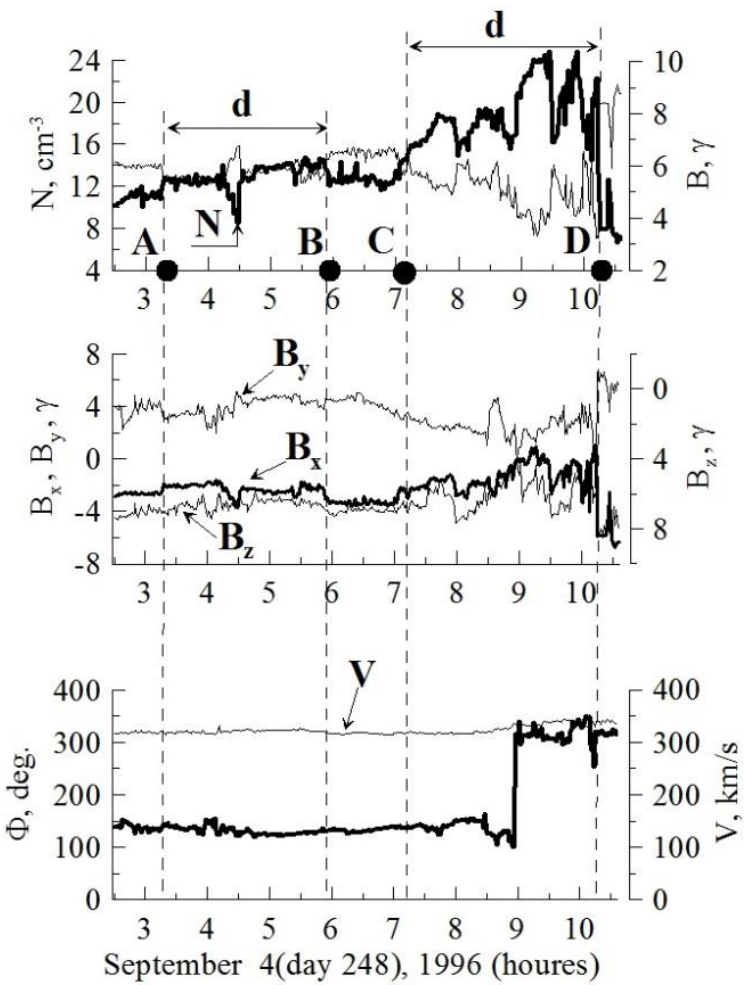

Figure 3. Solar wind parameters as a function of time on September 4, 1996 (day 248), as derived from Wind data. Plasma density $N(t)$ (thick curve), IMF modulus $B$ (thin curve) (top panel); $B_{x}, B_{y}, B_{z}$ (middle panel); IMF azimuth angle $\Phi$ (thick curve), SW plasma velocity $V$ (bottom panel). $N, V$, and $B$ are given with $\sim$-min resolution

The profile of $N(t)$ exhibits two humps of density ( $A B$ and $C D$ segments in the top panel of Figure 3 with different maximum values of $N$, which have an angular size $d \approx 1.5^{\circ}-1.8^{\circ}$. They have a smaller-scale internal structure. At boundaries of these humps (shown by vertical dashed lines), $B$ sharply increases, and $N$ decreases. $B(t)$ anticorrelates with $N(t)$. The anticorrelation coefficient in the time interval $2.5-10.8 \mathrm{hrs} K \approx-0.81$. No noticeable correlation or anticorrelation between $N(t)$ and $B_{x}(t), B_{y}(t), B_{z}(t)$ is seen. The velocity $V$ is virtually constant (up to at least $\pm 10 \%$ in the time interval of interest).

In oblique waves, the situation is intermediate. Since in our case $N(t)$ anticorrelates with $B(t)$, these are not magnetized plasma oscillations but spatial structures propagating from the Sun.

Direct evidence may be simultaneous measurements made by two spaced-apart spacecraft Wind and IMP-8 (Figure 4). For the September 4, 1996 event considered the mean coordinates (in units of the Earth radius) of SC relative to Earth's center were as follows: for Wind $x \approx 100$, $y \approx 0$; $z \approx 24$; for IMP-8 $x \approx 24, y \approx-27, z \approx-6$. Let us make a comparison for the first peak of $N$ (the $\mathrm{AB}$ segment in Figure 3) and a part of the second peak of $N$ (a part of the CD segment because we do not have IMP-8 data for the entire CD segment), using the magnetic field $B$ as an example because it is recorded with the best temporal resolution (by Wind with $\approx 3 \mathrm{~s}$, by IMP-8 with $\approx 12 \mathrm{~s}$ ). 

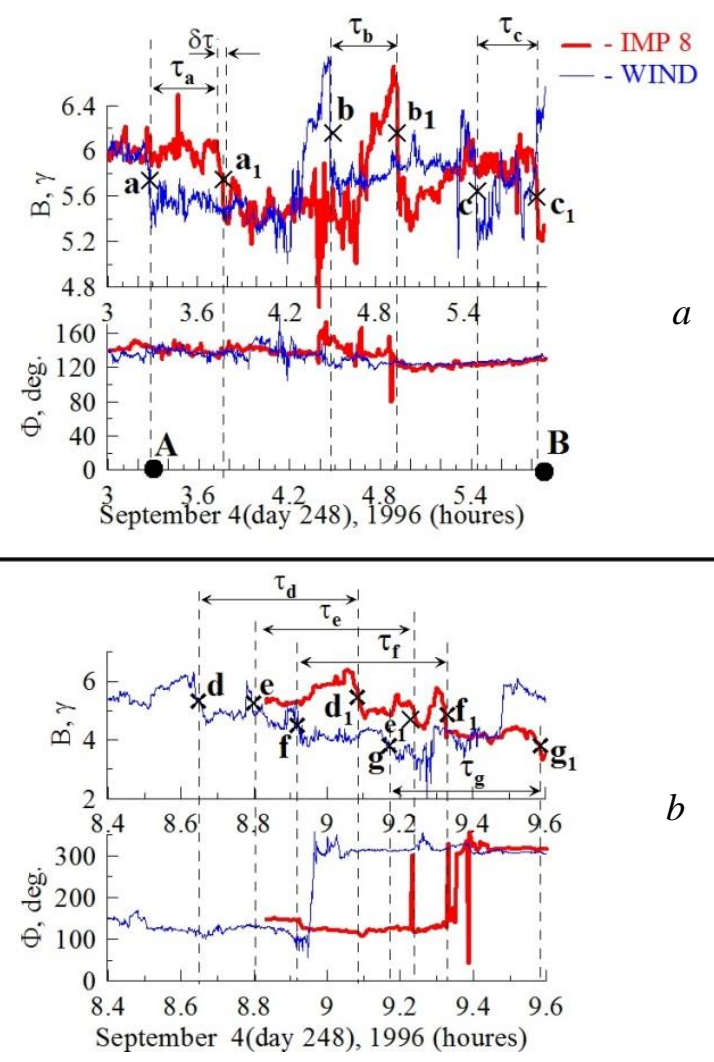

Figure 4. Modulus $B$ (top panels) and azimuth angle $\Phi$ (bottom panels) of the magnetic field as derived from measurements made by Wind (blue curves) and IMP-8 (red curves) on September 4, 1996 (day 248): for the AB segment in Figure $1(a)$; for a part of the CD segment in Figure $3(b)$

As characteristic points from which the movement of the structures between two SC is studied we select two midpoints of current sheets (magnetic field jumps): $\mathrm{a}$ and $\mathrm{a}_{1}, \mathrm{~b}$ and $\mathrm{b}_{1}, \mathrm{c}$ and $\mathrm{c}_{1}, \mathrm{~d}$ and $\mathrm{d}_{1}$ (Figure 4, $a$, top panel), and e and $\mathrm{e}_{1}, \mathrm{f}$ and $\mathrm{f}_{1}, \mathrm{~g}$ and $\mathrm{g}_{1}$ (Figure 4, $b$, top panel). The appearance of these layers in an IMP-8 signal is delayed by $\tau \approx 25-28 \mathrm{~min}$ with respect to the time of recording on Wind. The azimuth angle $\Phi$ in parts following each of the sheets remains virtually unchanged when moving from Wind to IMP-8 (Figure 4, $a, b$, bottom panels). The characteristic time scales $\delta \tau$ of the magnetic field jumps considered (Figure 4, $a$, top panel) whose minimum size can be a fraction of minute change slightly over $t>>\delta \tau$, i.e. we deal with propagation of quasi-static structures. Since current sheets bound areas with higher density and lower magnetic field (see, e.g., segments d in Figure 3), we deal with magnetic tubes: the diamagnetic current on their surface causes the field within the tubes to decrease. This agrees with the conclusions drawn in [Eselevich, Eselevich, 2006a, b] that structures inside HPS are manifestations of higher brightness rays (magnetic tubes) of the coronal streamer belt near the Sun. The angular size of the ray structures of the streamer belt when moving from the Sun to Earth's orbit remains constant, indicating their quasi-stationarity over $\approx 150$ million $\mathrm{km}$.

The anticorrelation between $N(t)$ and $B(t)$ in the absence of noticeable anticorrelation between $N(t)$ and individual components of IMF in HPS argues for the fact that these magnetic tubes are stretched along the direction of $\mathbf{B}$. As noted above, the $B$ weakening inside the tubes is a reflection of diamagnetism of highconductivity magnetized plasma with inhomogeneous boundaries [Frank-Kamenetsky, 1968]. A cause of the diamagnetism is a diamagnetic (or drift) current on boundary surfaces of tubes, which reduces $B$ inside the tube. In the case of equilibrium in the direction perpendicular to $\mathbf{B}$, the total pressure should remain constant $P=N\left(T_{\mathrm{e}}+T_{\mathrm{p}}\right)+B^{2} /(8 \pi)$. The fact that the total pressure in HPS remains constant has previously been noted in [Winterhalter et al., 1994]. As shown below, in most cases in different parts of HPS this condition holds (for the event of interest there is no data on $T_{\mathrm{e}}$ ).

At the same time, according to [Schwenn, March, 1991], there is developed Alfvén turbulence in HPS. The existence of quasi-static structures where the turbulence occurs can presumably be explained by the fact that the level of turbulent Alfvén oscillations $(\Delta B / B)_{\mathrm{T}}$ is much lower than the magnetic field modulus in quasistatic HPS structures $(\Delta B / B)_{\mathrm{K}} \sim 150-200 \%$. The turbulent oscillations may play a role of weak effective collisions that have no significant effect on quasi-static structures.

A surprising property of magnetic tubes in HPS is the presence of a fine structure in them on several spatial scales.

Let us consider the magnetic tube $\mathrm{AB}$ (see the notation in Figure 3 ) with an angular size $d \approx 1.5^{\circ}$ on a more extended time scale (Figure 5, top panel).

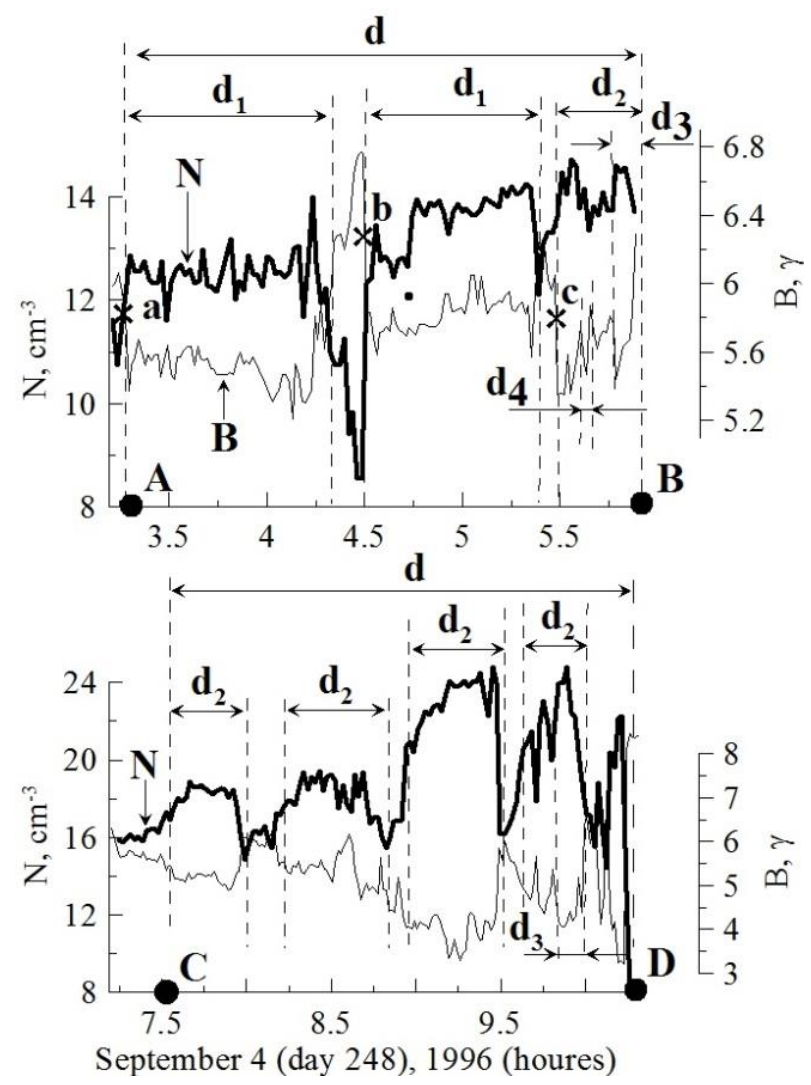

Figure 5. The same data as in Figure 3 (top panel) for parts of September 4, 1996 (day 248) for a more extended time scale: AB segment (top), CD segment (bottom). The time resolution is $3 \mathrm{~s}$ 
It, in turn, consists of two tubes with an angular size $d_{1} \approx 0.5^{\circ}-0.7^{\circ}$ and one tube with $d_{2} \approx 0.23^{\circ}$. The last tube includes two tubes with $d_{3} \approx 0.07^{\circ}$. The same anticorrelation between $N(t)$ and $B(t)$ is also seen on a yet smaller scale $d_{4} \approx 0.03^{\circ}$ (Figure 5, top panel). A similar situation also occurs in the CD segment (Figure 5, bottom panel). In it, the tube with $d \approx 1.5^{\circ}$ includes four tubes with $d_{2} \approx 0.2^{\circ}-0.3^{\circ}$. In this case, at least one of them (the last with $d_{2} \approx 0.2^{\circ}$ ) consists of three tubes with $d_{3} \approx 0.05^{\circ}$ $0.07^{\circ}$. As shown above, these tubes of various scales propagate from the Sun with approximately equal velocities, comprising a single HPS structure.

Thus, this analysis indicates the phenomenon of selfsimilarity (about similarity in the tube geometry see below) inherent in fractal structures [Milovanov, Zelenyi, 1994, 1999], namely, the properties of magnetic flux tubes corresponding to the minimum angular size $d_{4} \approx 0.03^{\circ}$ (Figure 5, top panel), the same as for the tubes with $d \approx 1.5^{\circ}-1.8^{\circ}$, i.e. they remain unchanged when the scale changes $\sim 50-60$ times.

By properties of the tubes on different spatial scales are meant (by the example of September 4, 1996) the following:

- diamagnetism (anticorrelation between an increase in $N(t)$ and a decrease in $B(t)$ );

- quasi-static nature, at least, for the time of their movement between Wind and IMP-8;

- approximate maintenance of the total pressure $P=N\left(T_{\mathrm{e}}+T_{\mathrm{p}}\right)+B^{2} /(8 \pi)$ in the direction perpendicular to $\mathbf{B}$;

- $\quad$ increased value of $\beta=8 \pi N\left(T_{\mathrm{e}}+T_{\mathrm{p}}\right) / B^{2}$ inside the tubes as compared to their surrounding plasma.

For September 4, 1996, the last two properties cannot be verified through lack of data on $T_{\mathrm{e}}$.

We therefore examine the HPS part in Earth's orbit in the second half of June 15, 1999 (day 166), for which $T_{\mathrm{e}}$ data is available. To this event near the Sun corresponds the streamer belt part crossing the plane of the ecliptic almost vertically (see NL on the synoptic map CR 1950 [http://wso.stanford.edu/] at a longitude $210^{\circ}$ $220^{\circ}$ (June 9-10, 1999)). Figure 6, $a$ shows that $B(t)$ and $N(t)$ are in antiphase (the anticorrelation coefficient $K \approx-0.8$ ). There are two regions of increased density $N_{\text {max }}>10 \mathrm{~cm}^{-3}$ with $d \approx 2.5^{\circ}$ and $d \approx 1.7^{\circ}$. These regions have a complex (with several IMF sign changes) sector boundary at the end of June 15 (Figure 6,c). $B(t)$ and $N(t)$ are in antiphase $(K \approx-0.8)$. The velocity $V$ (black circles) is almost constant throughout the interval of interest. Within these regions there is a smaller-scale structure $\left(N_{\max }>10 \mathrm{~cm}^{-3}\right)$ with a typical angular size $d_{1} \approx 0.6^{\circ}-0.8^{\circ}$. Inside the peaks with $d_{1}$ there are peaks with $d_{2} \approx 0.15^{\circ}-0.20^{\circ}$; and inside the latter, peaks with $d_{3} \approx 0.03^{\circ}-0.04^{\circ}$. This follows from Figure $7, a$, which shows the region with $d \approx 1.7^{\circ}$ on a more extended time scale. For each of these peaks of any of the said angular dimensions $d_{\mathrm{n}}, B(t)$ and $N(t)$ are antiphase (Figures 6, $a$ and $7, a), \beta$ in the vicinity of $N$ peaks noticeably exceeds that outside the peaks (Figures $6, b$ and $7, b$, thin line).

The total pressure $P=N\left(T_{\mathrm{e}}+T_{\mathrm{p}}\right)+B^{2} /(8 \pi)$, averaged over oscillations with a period of $\tau \leq 10 \mathrm{~min}$, change a little inside and outside the peaks (Figures $6, b$ and 7, $b$, thick curve). Throughout the interval of interest on the scale of peaks with $d \approx 1.7^{\circ}-2.5^{\circ}$, the total pressure also remains constant up to at least $\pm 15 \%$.

The current sheets associated with boundaries of the largest peaks $N_{\max }>10 \mathrm{~cm}^{-3}$ are labeled a, b, c, d, and $\mathrm{a}_{1}$, $\mathrm{b}_{1}, \mathrm{c}_{1}, \mathrm{~d}_{1}$ as recorded by Wind and IMP-8 respectively (Figures $6, d$ and $7, d$ ). Times of delay in passage of these structures between SC $\tau_{\mathrm{a}} \approx 58 \mathrm{~min}, \tau_{\mathrm{b}} \approx 53 \mathrm{~min}$, $\tau_{\mathrm{c}} \approx 54 \mathrm{~min}, \tau_{\mathrm{d}} \approx 52 \mathrm{~min}$, i.e they are close. This means that the entire set of structures moves as a single formation with nearly the same velocity. All this testifies to the fact that the peaks $N_{\max }>10 \mathrm{~cm}^{-3}$ with $d, d_{1}, d_{2}, d_{3}$, etc. are diamagnetic tubes, smaller tubes filling larger tubes.

The characteristic features of the behavior of HPS parameters in Earth's orbit determined from the two examples manifest themselves to a degree in any HPS part in the absence of sporadic streams. This conclusion has been drawn from the results of the study of $\sim 40$ events occurring in 1996-1999. It should be noted here that of particular importance for the concept of fractality is self-similarity between embedded diamagnetic tubes. Let us illustrate this for the HPS parts under study through the following considerations.

Let a large diamagnetic tube be filled with diamagnetic tubes of smaller transverse dimensions. Large and small tubes can be considered self-similar if their crosssections are similar in shape and close to circle. The following statements attest to the fact that in our case it should be so.

1. The tilt angles in the 40 HPS parts under study with respect to the plane of the ecliptic were different. This means that the magnetic tubes intersected SC at different arbitrary angles. And all of them gave approximately the same result: angular dimensions of embedded magnetic tubes vary from $d \approx 1.5^{\circ}-3.0^{\circ}$ to minimum $d \approx 0.03^{\circ}-0.06^{\circ}$ (Figure 5 , top panel). This indicates that cross-sections of the tubes are close to circle.

2. General physical grounds: it is unlikely that crosssections of diamagnetic tubes have the shape of ellipse or any other figure.

\section{LAYOUT OF DIAMAGNETIC TUBES IN THE HELIOSPHERE AND DETERMINATION OF VELOCITY OF THEIR MOVEMENT FROM THE SUN}

Based on the facts presented in the preceding section, the cross-section of the streamer belt and its extension in the heliosphere by the plane of the ecliptic can be schematically represented as a cross-section of diamagnetic tube by the plane of the ecliptic containing a moving plasma with density higher than the surrounding plasma. Near the Sun, the tube is oriented radially, and with distance from the Sun it gradually bends in the counter-rotational direction. Its angular size $d$ remains approximately constant all the way from the Sun to Earth. It is obvious that at any distance from the Sun the tube is oriented along IMF lines, which make up the tube 


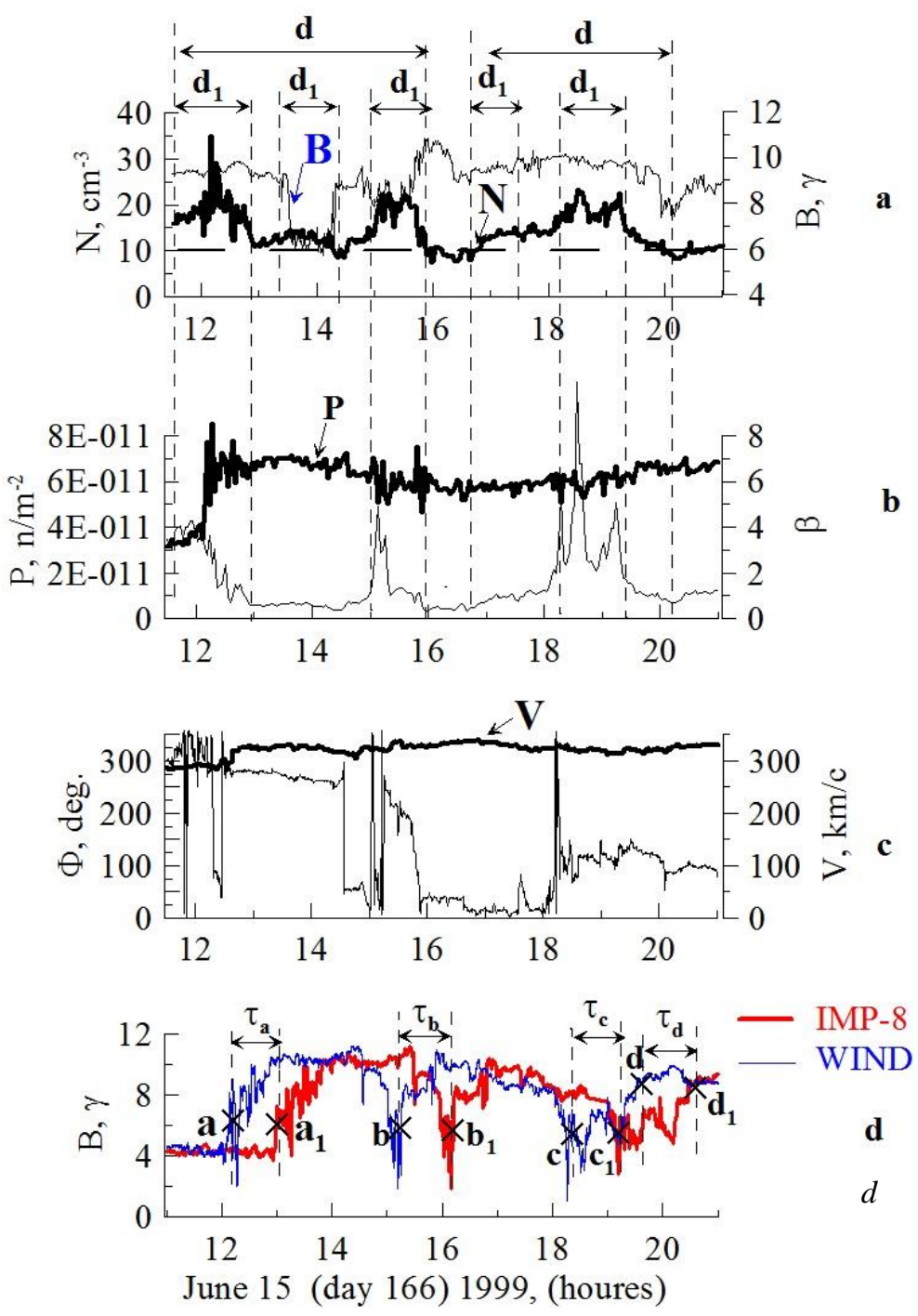

Figure 6. SW parameters as a function of time on June 15, 1999 (day 166): plasma density $N(t)$ (thick curve) $B$ (thin curve) with $\approx 1$-min temporal resolution $(a)$; total pressure $P=N\left(T_{\mathrm{e}}+T_{\mathrm{p}}\right)+B^{2} /(8 \pi)$ (thick curve) and $\beta=8 \pi\left[N\left(T_{\mathrm{e}}+T_{\mathrm{p}}\right)\right] / B^{2}$ (thin curve) with $\approx 2.5$-min temporal resolution $(b)$; azimuth angle $\Phi$ (thin curve) and SW $V$ (solid circles), with $\approx 1$-min temporal resolution as derived from Wind data $(c)$; magnetic field modulus $B$ as derived from measurements by Wind (blue curves, $\approx 3$-s resolution) and IMP-8 (red curves, $\approx 15$-s resolution) $(d)$

(curved lines with arrows bounding an area with dots in Figure 8). In Earth's orbit, the angle of inclination of the tube toward the OX axis as well as the line of its leading edge (thick line $\mathrm{AB}$ in Figure 8) is therefore equal to the azimuth angle $\Phi$ of IMF lines. Because of the increased plasma density inside, the tube is a spatial inhomogeneity with respect to its surrounding plasma. The SW plasma moves radially everywhere in the plane of the ecliptic both inside the inhomogeneity and outside it. The quasi-stationary pattern of such a radial SW stream with spatial irregularities in plasma density distribution in the plane of the ecliptic rotates with the Sun and has a linear velocity $V_{0}=2 \pi L / T=2 \pi 215 R_{\odot} / T \approx 405 \mathrm{~km} / \mathrm{s}$ in Earth's orbit, where $R_{\odot}=7 \cdot 10^{5} \mathrm{~km}$ is the solar radius, $T \approx 27$ days. Given the presence of these two independent movements in the plane of the ecliptic, we derive formulas for calculating the angle $\Phi_{\mathrm{C}}$ of inclination of the $\mathrm{AB}$ front of the tube with plasma of the slow SW having velocity $V$ in Earth's orbit, and the delay time $\tau_{\mathrm{C}}$ of passage of the $\mathrm{AB}$ front through Wind (point $\mathrm{A}$ ) and IMP-8 (point D), indicated in Figure 8.
Let the source of slow SW plasma at $t_{0}=0$ be on the central meridian of the Sun $\left(\varphi=0^{\circ}\right)$. Plasma particles emitted from the source at that moment move radially along the SA (or OX) line. At $t<<T$ due to the solar rotation, the source of the slow SW is at $\varphi<<360^{\circ}$ to the central meridian. Particles emitted by the source move radially along the SB line. From Figure 8 it follows that

$$
\tau_{C} \approx \frac{\varphi T}{2 \pi}+\frac{L-L_{1}}{V} \approx \frac{y-y_{1}}{V_{\mathrm{o}}}+\frac{x-x_{1}}{V} .
$$

It is considered here that $\Phi=2 \pi\left(y-y_{1}\right) / L$, where $x, y$ and $x_{1}, y_{1}$ are the coordinates (in terms of the sign) of IMP-8 (point D) and Wind (point A) respectively.

The angle $\psi$ of inclination of the $\mathrm{AB}$ front relative to the AC line (Figure 8 ) in view of the inequality $L-$ $L_{1}<<L$ is determined by the formula:

$$
\operatorname{tg} \psi=\frac{C B}{C A} \approx \frac{\varphi T V}{2 \pi} \frac{2 \pi}{\varphi L}=\frac{V}{V_{0}} .
$$

The desired azimuth angle $\Phi_{\mathrm{C}}$ is related to the angle $\psi$ by 


$$
\begin{aligned}
& \Phi_{\mathrm{C}}=90^{\circ} \psi \text { if } \Phi_{\mathrm{C}}<180^{\circ}, \\
& \Phi_{\mathrm{C}}=270^{\circ}+\psi \text { if } \Phi_{\mathrm{C}}>180^{\circ} .
\end{aligned}
$$

Since for HPS in Earth's orbit $V \approx 350 \mathrm{~km} / \mathrm{s} \pm 50$ $\mathrm{km} / \mathrm{s}$, for the quasi-stationary diamagnetic tube $\Phi_{\mathrm{C}} \approx$ $\approx 130^{\circ} \pm 5^{\circ}$ or $\Phi_{\mathrm{C}} \approx 310^{\circ} \pm 5^{\circ}$.

To verify the above picture, let us compare $\tau_{\mathrm{C}}, \Phi_{\mathrm{C}}$ calculated with $\tau_{\mathrm{m}}, \Phi_{\mathrm{m}}$ measured. Make this comparison, using the September 4, 1996 event considered as an example.

$\Phi_{\mathrm{m}}$ (for the current sheet a $\Phi_{\mathrm{m}}=\Phi_{\mathrm{a}}$, for the current sheet b $\Phi_{\mathrm{m}}=\Phi_{\mathrm{b}}$, etc.) were found from the formula $\Phi_{\mathrm{m}}$ $=\left(\Phi+\Phi_{1}\right) / 2$, where $\Phi$ and $\Phi_{1}$ are the azimuth angle averaged over the 3-min interval immediately before the arrival of the current sheet at Wind and IMP-8 respectively. $\Phi$ and $\Phi_{1}$ are usually close. In top panels of Figure $4, a, b$, the location of current sheets are marked with crosses with the corresponding letter. The time of movement of the sheet between the two SC are designated by $\tau$ with the index corresponding to the letter of the sheet. As $V$ we take the mean SW velocity on the way of each of the sheets moving between the two SC. The measured $V, \tau_{\mathrm{m}}, \Phi_{\mathrm{m}}$ are shown in table columns 9 ,
10,12 . Results of $\tau_{\mathrm{C}}, \Phi_{\mathrm{C}}$ calculated from Formulas (1)(4) for midpoints of current sheets (magnetic field jumps) recorded sequentially on Wind and IMP-8 (with index 1): $a$ and $a_{1}, b$ and $b_{1}, c$ and $c_{1}, d$ and $d_{1}$, e and $e_{1}, f$ and $\mathrm{f}_{1}, \mathrm{~g}$ and $\mathrm{g}_{1}$ in Figure 4, $a, b$ are shown in table columns 11 and 13 .

For the time interval of interest, the coordinates of Wind (table columns 3-5) and IMP-8 (columns 6-8) were respectively

Wind: $x \approx 100 R_{\mathrm{E}}, y \approx 0, z \approx-3 R_{\mathrm{E}}$;

IMP-8: $x \approx 24 R_{\mathrm{E}}, \mathrm{y} \approx-27 R_{\mathrm{E}}, z \approx-6 R_{\mathrm{E}}, \Phi_{\mathrm{m}}=\Phi_{\mathrm{a}}$.

Substituting the coordinates of the satellites and the values $V$ and $V_{0}$ for points a-g in Formulas (1)-(4), find $\tau_{\mathrm{C}}$ and $\Phi_{\mathrm{C}}$ for the sheets considered, shown in columns $11\left(\tau_{\mathrm{C}}\right)$ and $13\left(\Phi_{\mathrm{C}}\right)$. The results check well with the observed values. The mean delay time for all the seven sheets $\tau_{\mathrm{a}} \approx 25 \pm 1.3 \mathrm{~min}$. Thus, the velocity of all these sheets appears to be the same up to $\approx 5 \%$, i.e. the entire fractal structure propagates as a single formation.

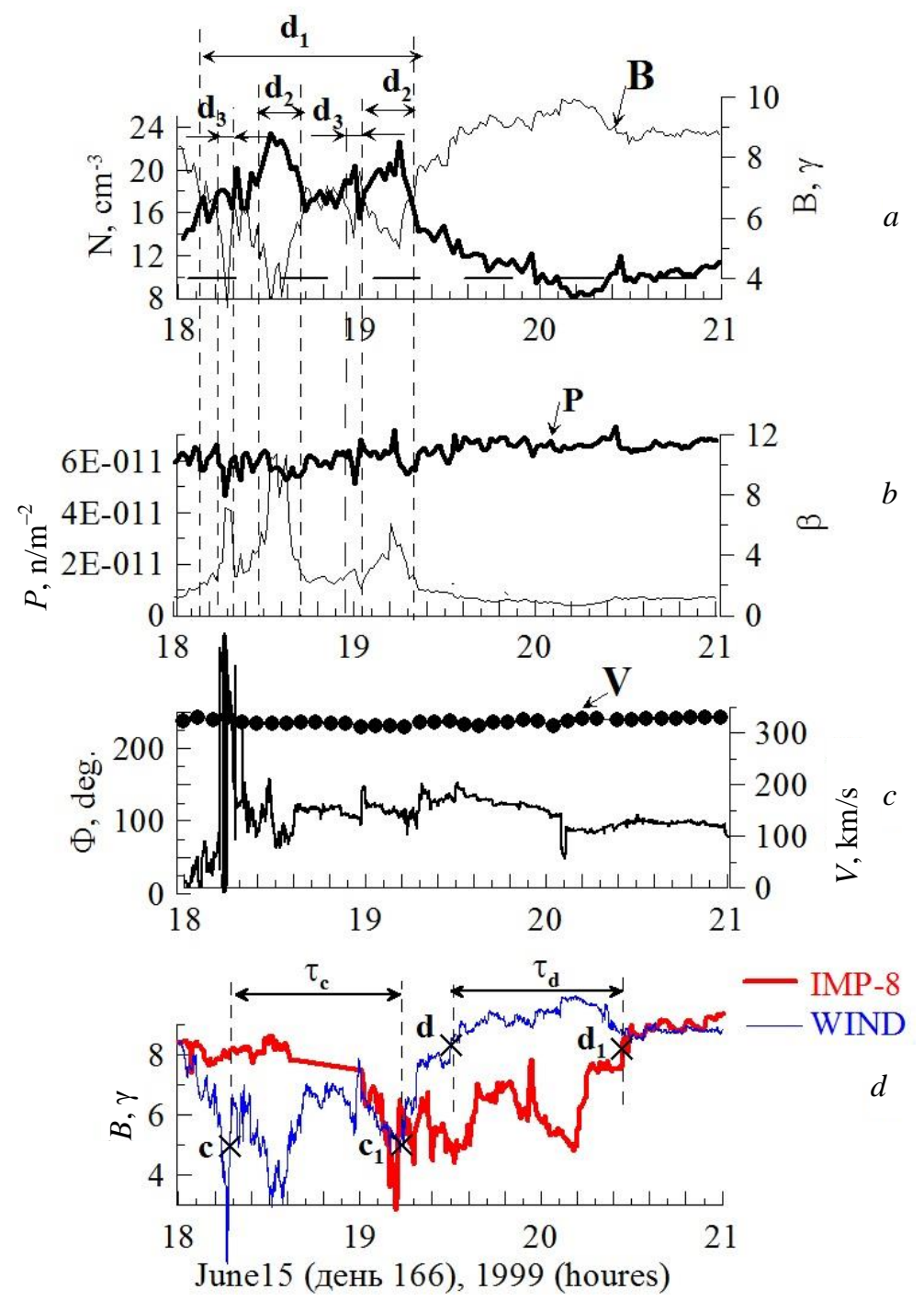

Figure 7. The same data as in Figure 6 for a part of June 15, 1999 (day 166) on a more extended time scale. Data in panels $a, b$ and velocities $V$ in panel $c$ are applied with $\approx 1$-min temporal resolution; all the others, with $\approx 15$-s resolution 


\section{Earth}

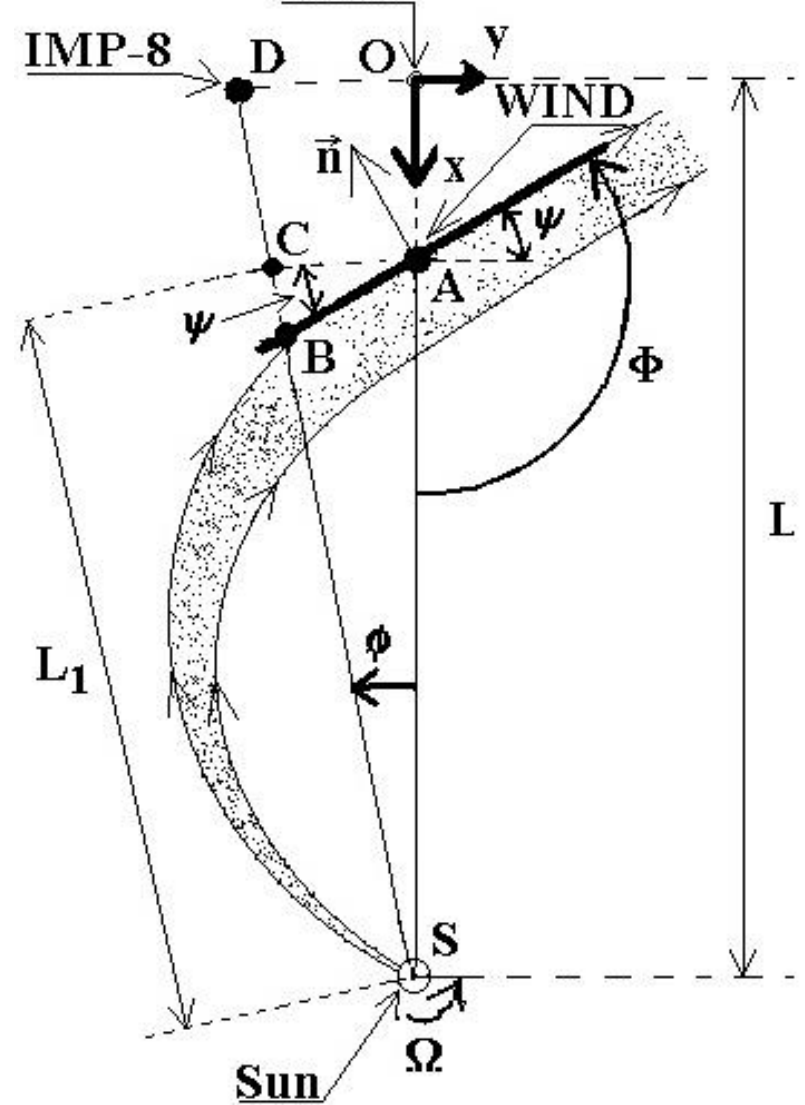

Figure 8. Cross-section of the streamer belt and its extension in the heliosphere by the plane of the ecliptic

In a similar manner, we have analyzed 18 events (see Table). The ratios $\tau_{\mathrm{m}} / \tau_{\mathrm{c}}$ as a function of the normalized distance between $\mathrm{SC} I / R_{\mathrm{E}}$ are shown in Figure 9.

Mean deviations from the lines $\tau_{\mathrm{m}} / \tau=1$ and $\Phi_{\mathrm{m}} / \Phi_{\mathrm{c}}=1$ are $\approx 15 \%$ and $\approx 5 \%$ respectively. Thus, the proposed picture of propagation of a quasi-stationary diamagnetic tube with plasma in SW fits the experiment reasonably well.

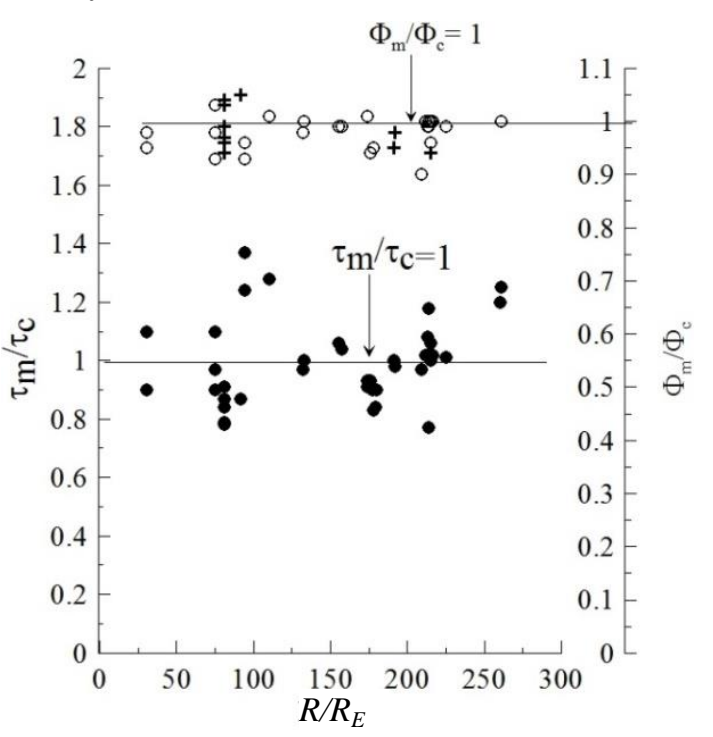

Figure 9. Ratios $\tau_{\mathrm{m}} / \tau_{\mathrm{c}}$ (solid circles) and $\Phi_{\mathrm{m}} / \Phi_{\mathrm{c}}$ (light circles indicate $\Phi_{\mathrm{m}}>180^{\circ}$; crosses, $\Phi_{\mathrm{m}}<180^{\circ}$ ) as a function of normalized distance $I / R_{\mathrm{E}}$ between $\mathrm{SC}$

\section{ANALYZING STABILITY \\ OF CURRENT AT BOUNDARIES OF DIAMAGNETIC TUBES}

As mentioned above, at boundaries of diamagnetic tubes there is a jump in the magnetic field modulus $\delta B$ on the spatial scale $\Delta$, and hence there is its associated diamagnetic (or drift) current. Figure 10 shows two examples of such jumps for two different events: September 4, 1996 and June 18, 1998

Let us focus on the latter event for which all parameters needed are known in the jump (point $\mathrm{C}$ ): $V \approx 350$ $\mathrm{km} / \mathrm{s}, \quad \Phi \approx 300^{\circ}, \quad \psi \approx 30^{\circ}, T_{\mathrm{e}} \approx 1.4 \cdot 10{ }^{5} \mathrm{~K}, \quad T_{\mathrm{p}} \approx 3 \cdot 10{ }^{4} \mathrm{~K}$, L $\quad N \approx 30 \mathrm{~cm}^{-3}, B \approx 11 \gamma=1.1 \cdot 10^{-4} \mathrm{G}$. First, estimate the current sheet thickness $\Delta$, knowing its time width $\delta t$.

The value $\Delta \approx V \cos \psi \delta t \approx 912 \mathrm{~km}$. Compare $\Delta$ with the ion Larmor radius $\rho_{\mathrm{i}} \approx v_{\mathrm{tp}} / \omega_{\mathrm{hp}}$. Find the thermal proton velocity $v_{\mathrm{tp}} \approx 25 \mathrm{~km} / \mathrm{s}$ and the Larmor proton frequency $\omega_{\mathrm{hp}} \approx 1.1 \mathrm{~s}^{-1}$. Hence, $\rho_{\mathrm{i}} \approx 23 \mathrm{~km}$. Thus, $\Delta \approx 40 \rho_{\mathrm{i}}$. This value should, however, be regarded as the upper limit of $\Delta$ since the observed minimum time width of the jump $\delta t$ is limited by $\sim 3$-s resolution of the measurement method, hence the true value of $\delta t$ should be lower.

Analyze the stability of the drift current at the tube boundary. It is known that the Buneman instability can develop if the following condition holds [Mikhailovsky, 1970]:
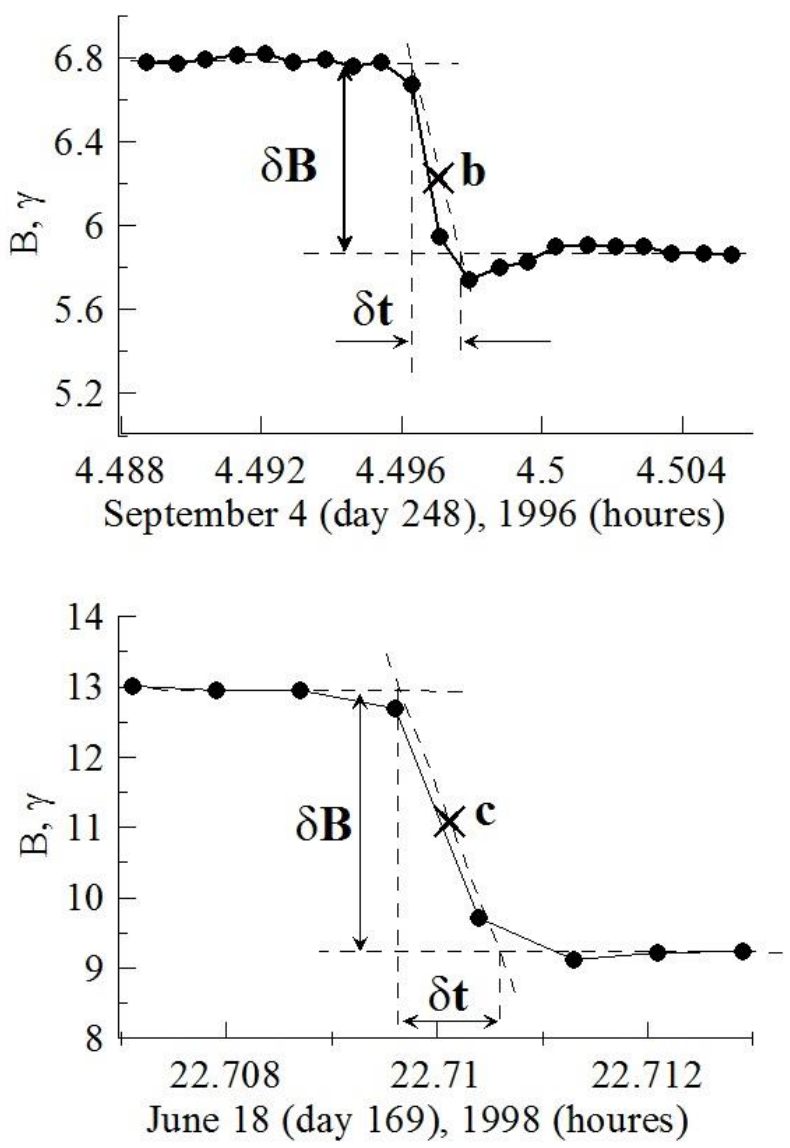

Figure 10. Examples of magnetic field jumps $B$ with maximum temporal resolution (dots are plotted with a 3-s interval) for two events in Earth's orbit as derived from Wind data: September 4 (day 248), 1996 (top); June 18 (day 169), 1998 (bottom) 


\begin{tabular}{|c|c|c|c|c|c|c|c|c|c|c|c|c|}
\hline \multirow[t]{3}{*}{ Day } & \multirow{3}{*}{$\begin{array}{c}t_{0} \\
\text { hours }\end{array}$} & \multicolumn{6}{|c|}{ SC coordinates } & \multirow{3}{*}{$\begin{array}{c}V, \\
\mathrm{~km} / \mathrm{s}\end{array}$} & \multirow{3}{*}{$\begin{array}{l}\tau_{\mathrm{m}} \\
\min \end{array}$} & \multirow{3}{*}{$\begin{array}{c}\tau_{c} \\
\min \end{array}$} & \multirow{3}{*}{$\begin{array}{l}\Phi_{\mathrm{m}}, \\
\text { deg. }\end{array}$} & \multirow{3}{*}{$\begin{array}{l}\Phi_{\mathrm{C}} \\
\mathrm{deg}\end{array}$} \\
\hline & & \multicolumn{3}{|c|}{ Wind } & \multicolumn{3}{|c|}{ IMP-8 } & & & & & \\
\hline & & $x / R_{\mathrm{E}}$ & $y / R_{\mathrm{E}}$ & $z / R_{\mathrm{E}}$ & $x / R_{\mathrm{E}}$ & $y / R_{\mathrm{E}}$ & $z / R_{\mathrm{E}}$ & & & & & \\
\hline $\begin{array}{l}\text { May } 19,1996 \\
\quad(\text { day } 140)\end{array}$ & $\begin{array}{l}7.50 \\
7.96\end{array}$ & 102 & -34 & 4 & -21 & 10 & -16 & $\begin{array}{l}327 \\
327\end{array}$ & $\begin{array}{l}27 \\
28\end{array}$ & $\begin{array}{l}28 \\
28\end{array}$ & $\begin{array}{l}307 \\
310\end{array}$ & $\begin{array}{l}309 \\
309\end{array}$ \\
\hline $\begin{array}{l}\text { July } 15,1996 \\
\text { (day } 197 \text { ) }\end{array}$ & $\begin{array}{l}13.50 \\
14.70 \\
16.75\end{array}$ & 195 & 13 & -4 & 25 & -18 & 5 & 380 & $\begin{array}{l}47 \\
48 \\
51\end{array}$ & $\begin{array}{l}57 \\
57 \\
57\end{array}$ & 300 & 315 \\
\hline $\begin{array}{c}\text { September 04, } 1996 \\
\text { (day 248) }\end{array}$ & $\begin{array}{l}3.30 \\
4.50 \\
5.47 \\
8.64 \\
8.80 \\
8.92 \\
9.16\end{array}$ & 100 & 0 & 24 & 24 & -27 & -6 & $\begin{array}{l}320 \\
320 \\
320 \\
340 \\
340 \\
340 \\
340\end{array}$ & $\begin{array}{l}28 \\
27 \\
24 \\
26 \\
26 \\
26 \\
25\end{array}$ & $\begin{array}{l}31 \\
31 \\
31 \\
30 \\
30 \\
30 \\
30\end{array}$ & $\begin{array}{l}134 \\
132 \\
127 \\
122 \\
125 \\
125 \\
125\end{array}$ & $\begin{array}{l}128 \\
128 \\
128 \\
130 \\
130 \\
130 \\
318\end{array}$ \\
\hline $\begin{array}{c}\text { November 03, } 1996 \\
\text { (day 308) }\end{array}$ & 19.4 & 114 & -32 & 5 & 6 & -27 & -16 & 325 & 46 & 36 & 317 & 308 \\
\hline $\begin{array}{c}\text { November 04, } 1996 \\
\text { (day 309) }\end{array}$ & $\begin{array}{l}1.44 \\
2.41\end{array}$ & 110 & -26 & 6 & 20 & -15 & -18 & $\begin{array}{l}360 \\
375\end{array}$ & $\begin{array}{l}31 \\
33\end{array}$ & $\begin{array}{l}25 \\
24\end{array}$ & $\begin{array}{l}300 \\
290\end{array}$ & $\begin{array}{l}312 \\
313\end{array}$ \\
\hline $\begin{array}{c}\text { April 04, } 1997 \\
\text { (day 94) }\end{array}$ & 0.50 & 220 & 5 & & 13 & -23 & 18 & 410 & 60 & 62 & 286 & 314 \\
\hline $\begin{array}{c}\text { September 03,1997 } \\
\text { (day 246) }\end{array}$ & 15.60 & 80 & 33 & 0 & 23 & -25 & -15 & 390 & 21.6 & 27.8 & 131 & 135 \\
\hline $\begin{array}{c}\text { February } 23,1997 \\
(\text { day } 54)\end{array}$ & 9.74 & 210 & 15 & & -10 & -30 & -15 & 480 & 61 & 60 & 315 & 318 \\
\hline $\begin{array}{l}\text { March 02, } 1998 \\
\quad(\text { day 61) }\end{array}$ & $\begin{array}{l}6.60 \\
8.15\end{array}$ & & & & & & & $\begin{array}{l}400 \\
420\end{array}$ & $\begin{array}{l}66 \\
64\end{array}$ & $\begin{array}{l}55 \\
51\end{array}$ & $\begin{array}{l}315 \\
316\end{array}$ & $\begin{array}{c}- \\
315\end{array}$ \\
\hline $\begin{array}{l}\text { March 10, } 1998 \\
\quad(\text { day 69) }\end{array}$ & $\begin{array}{c}5.30 \\
8.50 \\
12.00\end{array}$ & 233 & -21 & -23 & 24 & 15 & 14 & $\begin{array}{l}320 \\
370 \\
430\end{array}$ & $\begin{array}{l}71 \\
54 \\
51\end{array}$ & $\begin{array}{l}60 \\
51 \\
51\end{array}$ & $\begin{array}{c}- \\
120 \\
305\end{array}$ & $\begin{array}{c}- \\
127 \\
317\end{array}$ \\
\hline $\begin{array}{l}\text { March 21, } 1998 \\
\quad(\text { day 80) }\end{array}$ & $\begin{array}{c}11.4 \\
12.55\end{array}$ & 232 & -24 & -15 & 20 & 0 & -15 & $\begin{array}{l}400 \\
420\end{array}$ & $\begin{array}{l}54 \\
36\end{array}$ & $\begin{array}{l}50 \\
47\end{array}$ & $\begin{array}{l}310 \\
316\end{array}$ & $\begin{array}{l}315 \\
316\end{array}$ \\
\hline $\begin{array}{c}\text { April 16, } 1998 \\
\text { (day 106) }\end{array}$ & $\begin{array}{l}20.25 \\
21.20 \\
\end{array}$ & 223 & -13 & 13 & 32 & 0 & 15 & $\begin{array}{l}350 \\
350 \\
\end{array}$ & $\begin{array}{l}55 \\
54 \\
\end{array}$ & $\begin{array}{l}55 \\
55 \\
\end{array}$ & $\begin{array}{l}125 \\
128 \\
\end{array}$ & $\begin{array}{l}131 \\
131 \\
\end{array}$ \\
\hline $\begin{array}{c}\text { June } 18,1998 \\
\text { (day 169) }\end{array}$ & $\begin{array}{c}4.0 \\
9.4 \\
22.7\end{array}$ & 77 & 62 & 14 & 35 & 0 & -15 & $\begin{array}{l}340 \\
340 \\
340 \\
\end{array}$ & $\begin{array}{l}29 \\
33 \\
27 \\
\end{array}$ & $\begin{array}{l}30 \\
30 \\
30 \\
\end{array}$ & $\begin{array}{l}325 \\
300 \\
310 \\
\end{array}$ & $\begin{array}{l}317 \\
317 \\
317 \\
\end{array}$ \\
\hline $\begin{array}{c}\text { July } 10,1998 \\
\text { (day 191) }\end{array}$ & 22.85 & 80 & 15 & 3 & 0 & -28 & 16 & 325 & 33 & 37 & 135 & 129 \\
\hline $\begin{array}{c}\text { June } 01,1999 \\
\text { (day 152) }\end{array}$ & $\begin{array}{l}13.60 \\
15.10\end{array}$ & 178 & -21 & -12 & 23 & -23 & 2 & $\begin{array}{l}350 \\
350\end{array}$ & $\begin{array}{l}50 \\
49\end{array}$ & $\begin{array}{l}47 \\
47\end{array}$ & $\begin{array}{l}311 \\
311 \\
\end{array}$ & $\begin{array}{l}315 \\
315\end{array}$ \\
\hline $\begin{array}{l}\text { June } 08,1999 \\
\quad(\text { day } 159)\end{array}$ & $\begin{array}{c}1.65 \\
5.60 \\
8.05 \\
10.20\end{array}$ & 195 & -21 & -10 & -11 & 31 & -11 & $\begin{array}{l}345 \\
360 \\
360 \\
365\end{array}$ & $\begin{array}{l}51 \\
51 \\
51 \\
51\end{array}$ & $\begin{array}{l}50 \\
50 \\
50 \\
50\end{array}$ & $\begin{array}{l}310 \\
310 \\
132 \\
132\end{array}$ & $\begin{array}{l}310 \\
312 \\
132 \\
132\end{array}$ \\
\hline $\begin{array}{l}\text { June } 15,1999 \\
\quad(\text { day } 166)\end{array}$ & $\begin{array}{l}12.50 \\
15.90 \\
20.20 \\
22.30\end{array}$ & 205 & -21 & -8 & 33 & -13 & 16 & $\begin{array}{l}290 \\
330 \\
330 \\
330 \\
\end{array}$ & $\begin{array}{l}58 \\
53 \\
54 \\
52\end{array}$ & $\begin{array}{l}62 \\
58 \\
58 \\
58\end{array}$ & $\begin{array}{l}310 \\
120\end{array}$ & $\begin{array}{l}306 \\
128\end{array}$ \\
\hline $\begin{array}{c}\text { September 26, } 1999 \\
\text { (day 269) }\end{array}$ & $\begin{array}{l}15.00 \\
20.25\end{array}$ & 61 & 13 & 3 & 33 & 4 & -5 & $\begin{array}{l}400 \\
460\end{array}$ & $\begin{array}{c}8 \\
10\end{array}$ & $\begin{array}{c}10 \\
9\end{array}$ & $\begin{array}{l}300 \\
310\end{array}$ & $\begin{array}{l}315 \\
317\end{array}$ \\
\hline
\end{tabular}


$v_{\mathrm{d}}>v_{\mathrm{tp}}$

Estimate $v_{\mathrm{d}}$ from the relation:

$$
v_{d} \approx \frac{\delta B c}{4 \pi e \Delta} \approx 0.54 \mathrm{~km} / \mathrm{s},
$$

where $c=3 \cdot 10^{5} \mathrm{~km} / \mathrm{s}, \quad e=4.8 \cdot 10^{-10} \quad$ CGSE units, $\delta B \approx 3 \cdot 10^{-5} \mathrm{G}$.

Hence, $v_{\mathrm{d}} \approx 0.54 \mathrm{~km} / \mathrm{s}<<v_{\mathrm{tp}} \approx 25 \mathrm{~km} / \mathrm{s}$.

Thus, the observed drift currents at the tube boundaries are resistant to excitation of electrostatic and electromagnetic oscillations of various types. This fact perhaps explains the existence of the traveling quasistationary diamagnetic tubes with higher density plasma in SW.

The fractal structure of SW diamagnetic tubes is shown in Figure 11. A similar structure for SW has been theoretically obtained by Milovanov and Zelenyi, [1994, 1999].

In what follows, instead of the term "diamagnetic tubes" we use a more general term "diamagnetic structures" (DS). It has been introduced and used for analyzing interaction of HPS parts with Earth's magnetosphere in [Parkhomov et al., 2018].

\section{COMPARING OBSERVATIONS OF SW DIAMAGNETIC PLASMOID AND HPS DIAMAGNETIC STRUCTURES}

Let us address the main problem of the above review of works on the structure of the slow SW that is still unsolved. It concerns the understanding of morphology and origin of the previously detected features in the slow SW: number density structures on time scales $\Delta t \approx 2 \div 80 \mathrm{~min}$ [Stansby, Horbury, 2018] and SW diamagnetic plasmoids on $\Delta t \approx 5 \div 10 \mathrm{~min}$ [Karlsson et al., 2015]. As noted in Introduction, number density structures were recorded had a size close to that of the plasmoids, and were recorded in the slow SW. Hereinafter, we therefore call structures of both the types SW diamagnetic plasmoids.

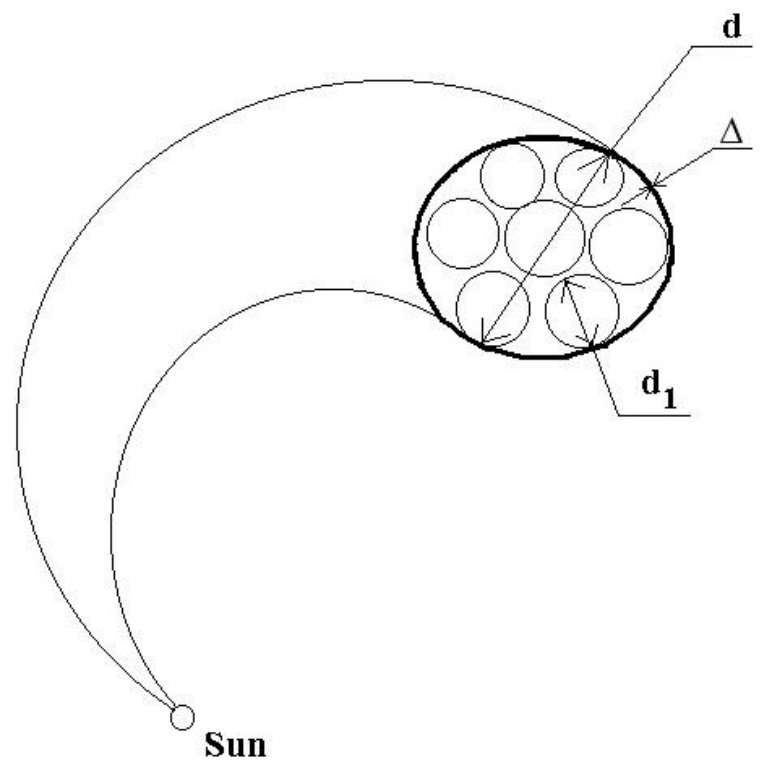

Figure 11. Scheme of the fractal structure of diamagnetic tube in SW derived from the results of this work at distances of $\leq 0.5$ AU [Stansby, Horbury, 2018]. They, like SW diamagnetic plasmoids, were diamagnetic,

Consider a typical HPS part in Earth's orbit on April 05, 2006 (day 96) at 04:35-04:55 UT.

Referring to Figure 12, $a$, the $B(t)$ and $N(t)$ profiles are in antiphase $(K \approx-0.8)$. The HPS part is composed of three diamagnetic structures with increased density $N_{\max }>(10 \pm 2) \mathrm{cm}^{-3}$, two of which have an angular size $d \approx 2^{\circ}$; and one, $d_{1} \approx 0.7^{\circ}$. The sector boundary is located at the beginning of the first part $d$ at $3.7 \mathrm{hr}$ on June 5 (b). $V$ remains virtually unchanged throughout the interval of interest. Within these three diamagnetic structures there are density increases (internal DS) of various smaller scales reflecting DS fractality of the slow SW.

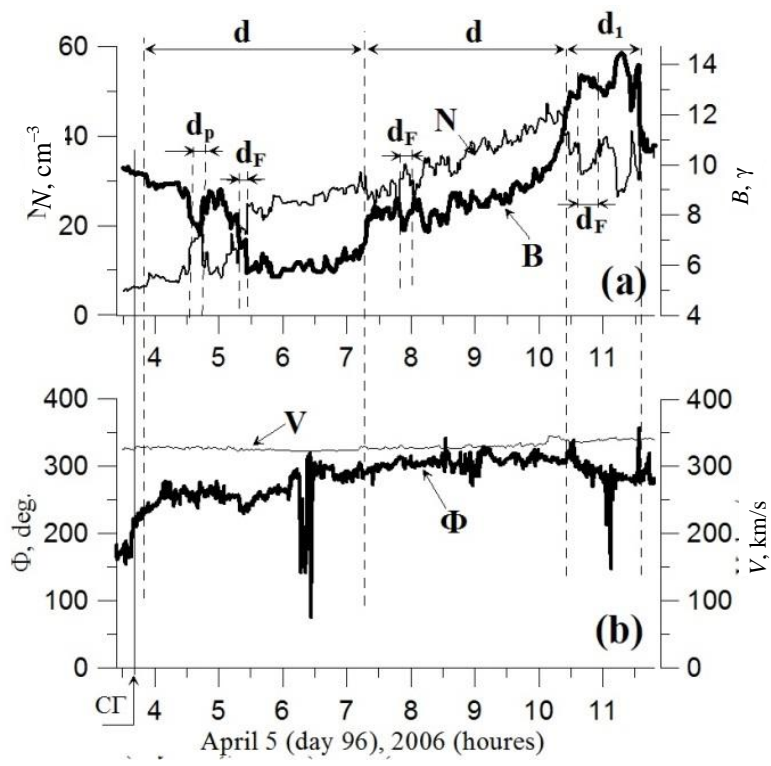

Figure 12. SW parameters as a function of time on April 05, 2006: plasma density $N(t)$ (thick curve), $B$ (thin curve) with $\approx 1$-min temporal resolution $(a)$; azimuth angle $\Phi$ (thick curve) and solar wind velocity $V$ (thin curve) with $\approx 1$-min temporal resolution $(b)$. Wind data. The position of the sector boundary is shown on the left by the vertical line with an arrow at the bottom and a mark $\mathrm{C} \Gamma$

Several of these DSs are indicated by horizontal arrows and designated by $d_{\mathrm{F}}$ in Figure 12, $a$. The SW diamagnetic plasmoid studied by [Karlsson et al. [2015] and labelled $d_{\mathrm{F}}$ is most likely to be one of these internal elements of diamagnetic structure fractality with an angular size $d \approx 2^{\circ}$ of the HPS part considered.

A similar comparative analysis carried out for another two SW diamagnetic plasmoids in [Karlsson et al., 2015] for April 04, 2004 and March 11, 2003 also leads to the same conclusion.

Thus, we can state with reasonable certainty that in the absence of sporadic streams SW diamagnetic plasmoids, examined by Karlsson et al. [2015], are internal elements of fractal diamagnetic structures of the slow SW.

\section{CONCLUSIONS}

1. A source of the quasi-stationary slow SW on the Sun is streamer belt and chains. The streamer belt encircles the Sun as a wave-like surface (skirt) and represents a sequence of pairs of rays with increased brightness 
(plasma density) or two closely spaced lines of rays. The neutral line of the radial component of the global solar magnetic field goes between rays of each pair along the belt. The streamer belt extension in the heliosphere is the heliospheric plasma sheet (HPS).

2. Higher-brightness rays of the streamer belt in Earth $\ni$ s orbit, i.e. in HPS, are diamagnetic tubes with higher plasma density $\left(N_{\max }>(10 \pm 2) \mathrm{cm}^{-3}\right)$ and lower interplanetary magnetic field strength. They also feature a fine structure on several spatial scales (fractality) from $d \approx 1.5^{\circ}-3.0^{\circ}$ to minimum $\mathrm{d} \approx 0.03^{\circ}-0.06^{\circ}$, i.e. the angular size of embedded tubes varies by almost two orders of magnitude.

3. Magnetic tubes on each observed spatial scale are diamagnetic, i.e. the diamagnetic current that decreases the magnetic field inside the tube and increases it outside the tube flows on their surface. The value $\beta=8 \pi\left[N\left(T_{\mathrm{e}}+T_{\mathrm{p}}\right)\right] / B^{2}$ inside the tube exceeds $\beta$ outside it.

4. In many cases, the total pressure $P=N\left(T_{\mathrm{e}}+T_{\mathrm{p}}\right)+B^{2} /(8 \pi)$ is almost constant in the tubes and outside them on any above scales.

5. Magnetic tubes are quasi-static structures and remain virtually unchanged when HPS moves between two spaced-apart SC. The fact that the angular size of HPS tubes when they move from the Sun to Earth's orbit remains constant indicates their quasi-stationary throughout the way.

6. The drift (or diamagnetic) current at the tube boundary is resistant to excitation of chaotic oscillations in a magnetized plasma. All this allows us to introduce a more common term for these tubes - diamagnetic structures (DSs) forming the basis of the slow SW or HPS in Earth's orbit.

These six points of the conclusion reflect foundations of the modern understanding of the nature and structure of the slow SW all the way from the Sun to Earth's orbit. The comparative analysis carried out at the end of this article, which is based on these conclusions, allowed us to figure out the morphology and origin of the new term "SW diamagnetic plasmoids" (or local enhancements of density), which appeared in a series of articles in the past decade. The new finding obtained from the analysis is formulated as follows.

7. The SW diamagnetic plasmoids discovered and studied in [Karlsson et al., 2015; Stansby, Horbury, 2018] represent a small-scale (in time and space) component of fractal diamagnetic structures of the slow SW.

I am grateful to Yu.I. Ermolaev who initiated this work. I also thank LASCO/SOHO, Wind, IMP-8 teams for providing data used in this paper.

The work was carried under project No. 007-0016318-00 of January 12, 2018.

\section{REFERENCES}

Borrini G., Wilcox J.M., Gosling J.T., Feldman W.C. Wilcox J.M. Solar wind helium and hydrogen structure near the heliospheric current sheet; a signal of coronal streamer at 1 AU. $J$. Geophys. Res. 1981, vol. 86, pp. 4565.

Eselevich V.G., Fainshtein V.G. The heliospheric current sheet (HCS) and high-speed solar wind: interaction effects. Planetary Space Sci. 1991, vol. 39, pp. 737-744.

Eselevich V.G., Fainshtein V.G. On the existence of the heliospheric current sheet without a neutral line. Planetary Space Sci. 1992, vol. 40, pp. 105.

Eselevich M.V., Eselevich V.G. Some features of coronal streamer belt in the solar corona and in Earth's orbit. Astronomicheskii Zhurnal [Astron. J.]. 2006a, vol. 83, no. 9, pp. 837-852. (In Russian).

Eselevich M.V., Eselevich V.G. Manifestation of radial structure of the coronal streamer belt as sharp peaks in the solar wind plasma density in Earth's orbit. Geomagnetism and Aeronomy. 2006b, vol. 46, iss.6, pp. 710-782. DOI: 10.1134/ S0016793206060132.

Eselevich V.G., Fainshtein V.G., Rudenko G.V. Study of the structure of streamer belts and chains in the solar corona. Solar Phys. 1999, vol. 188, pp. 277.

Eselevich V.G., Fainshtein V.G., Eselevich M.V. The existence of long-lived rays of the coronal streamer belt. Radial density and velocity distributions of the solar wind flowing in them. Solar Phys. 2001, vol. 200, pp. 259.

Eselevich M.V., Eselevich V.G. The double structure of the coronal streamer belt. Solar Phys. 2006, vol. 235, iss. 1-2, pp. 331-344.

Eselevich M., Eselevich V., Fujiki K. Streamer belt and chains as the main sources of quasi-stationary slow solar wind. Solar Phys. 2007, vol. 240, pp. 135-151. DOI: 10.1007/ s11207-006-0197-z.

Frank-Kamenetsky D.A. Lectures on Plasma Physics. Moscow, Atomizdat Publ., 1968. 288 p. (In Russian).

Howard R.A., Koomen M.J., Michels D.J., Tousey R., Detwiler C.R., Roberts D.E., et al. Report UAG-48. Synoptic observation of the solar corona during Carrington rotations 1580-1596 (11 October 1971 - 15 January 1973), World Data Center A for STP, NOAA, Boulder, Colorado, 1975.

Illing R.M., Hundhausen A.J. Disruption of a coronal streamer by an eruptive prominence and a coronal mass ejection. J. Geophys. Res. 1986, vol. 91, pp. 10951.

Ivanov K., Bothmer V., Cargill P.J., P., Kharshiladze A.F., Romashets E.P., Veselovsky I.S. Subsector structure of the interplanetary space. Proc. The Second Solar Cycle and Space Whether Euroconference. Vicvo Equense (Italy). 2002, pp. 317-320.

Karlsson, T., Brenning N., Nilsson H., Trotignon J.-G., Vallières X., Facsko G.. Localized density enhancements in the magnetosheath: Three-dimensional morphology and possible importance for impulsive penetration. J. Geophys. Res. 2012, vol. 117, iss. A3, pp., CiteID A03227. DOI: 10.1029/ 2011JA017059.

Karlsson T., Kullen, A., Liljeblad E., Brenning N., Nilsson H., Gunell H., Hamrin M. On the origin of magnetosheath plasmoids and their relation to magnetosheath jets. J. Geophys. Res.: Space Phys. 2015, vol. 120, iss. 9, pp. 73907403. DOI: $10.1002 / 2015 J A 021487$.

Korzhov N.P. Large-scale three-dimensional structure of the interplanetary magnetic field. Solar Phys. 1977, vol. 55, pp. 505.

Mikhailovsky A.B. Teoriya plazmennykh neustoichivostei [Theory of Plasma Instabilities]. Moscow, Atomizdat, 1970. vol. 1. 294 p. (In Russian).

Milovanov A.V., Zelenyi L.M. Fractal clusters in the solar wind. Adv. Space Res. 1994, vol. 14, pp. 123-133.

Milovanov A.V., Zelenyi L.M. Fraction excitations as a driving mechanism for the self-organized dynamical structuring in the solar wind. Astrophys. Space Sci. 1999, vol. 264, pp. 317-345.

Parkhomov V.A., Borodkova N.L., Eselevich V.G., Eselevich M.V., Dmitriev A.V., Chilikin V.E. Features of the impact of the solar wind diamagnetic structure on Earth's magnetosphere. Solar-Terrestrial Phys. 2017, vol. 3, no. 4, pp. 44-57. DOI: 10.12737/stp-34201705. 
Parkhomov V.A., Borodkova N.L., Eselevich V.G., Eselevich M.V., Dmitriev A.V., Chilikin V.E. Solar wind diamagnetic structures as a source of substorm- like disturbances. J. Atmosph. Solar-Terr. Phys. 2018, vol. 181, pp. 55-67. DOI: 10.1016/j.jastp.2018.10.010.

Schwenn R., March E. Physics of the inner heliosphere. II. Particle, waves and turbulence. Springer-Varlag, 1991, 185 p.

Stansby D., Horbury T.S. Number density structures in the inner heliosphere. Astron. Astrophys. 2018, vol. 613, N A62, pp. 7. DOI: 10.1051/0004-6361/201732567.

Svalgaard L.J., Wilcox W., Duvall T.L. A model combining the solar magnetic field. Solar Phys. 1974, vol. 37, pp. 157.

Wang Y.M., Sheeley N.R., Rich N.B. Coronal pseudostreamers. Astrophys. J. 2007, vol. 685, pp. 1340.

Winterhalter D., Smith E.J., Burton M.E., Murphy N. The heliospheric plasma sheet. J. Geophys. Res. 1994, vol. 99, pp. 6667.

Woo R., Armstrong J.W., Bird M.K., Patzold M. Finescale filamentary structure in coronal streamers. Astrophys. $J$. 1995, vol. 449, pp. L91-L94.

URL: http://wso.stan-ford.edu

How to cite this article

Eselevich V.G. Diamagnetic structures as a basis of quasistationary slow solar wind. Solar-Terrestrial Physics. 2019. Vol. 5. Iss. 3. P. 29-41. DOI: $10.12747 /$ stp-53201904 